\title{
Does variation in NIPA2 contribute to genetic generalized epilepsy?
}

${ }^{1}$ Epilepsy Research Centre, Department of Medicine, University of Melbourne, Austin Health, Heidelberg, Victoria 3084, Australia

${ }^{2}$ Florey Institute and Department of Paediatrics, University of Melbourne, Royal Children's Hospital, Parkville, Victoria 3052, Australia

${ }^{3}$ Corresponding author

\section{Correspondence To:}

Professor Samuel F. Berkovic

Address: Epilepsy Research Centre, Level 2, Melbourne Brain Centre, 245 Burgundy St. Heidelberg, Victoria 3084, Australia

Telephone: +61 39035 7093; Fax: +61 39496 2291; E-mail Address: s.berkovic@unimelb.edu.au

Running Title: NIPA2 and epilepsy

Key Words: NIPA2; genetic generalized epilepsy; deletion; insertion

Number of Text Pages: 4

Word Count: 525 
In their original investigation Jiang and colleagues reported three NIPA2 point mutations or indels from a cohort of 380 Han Chinese patients with childhood absence epilepsy (CAE), a major syndrome of genetic generalized epilepsy (GGE) (Jiang et al. 2012). These three novel variants included two missense changes (p.I178F and p.N244S) and a small insertion (p.N334_E335insD), that were absent in 700 control individuals.

We attempted to replicate these findings in our cohort of 494 Caucasian patients with GGE and over 130 Caucasian controls from the Australian population. Of the patients, 164 were diagnosed with CAE and the remaining 330 patients were diagnosed with generalized tonic clonic seizures (GTCS), juvenile absence epilepsy (JAE) or juvenile myoclonic epilepsy (JME). The coding regions and splice sites of the NIPA2 gene were screened in this cohort by PCR and Sanger sequencing using genespecific primers and standard methods.

Three rare variants were identified including the previously reported p.N334_335EinsD insertion (Table 1) (Jiang et al. 2012). We found the p.N334_E335insD insertion in 1.4\% of our patient cohort $(n=7 / 494)$ with a range of GGE subsyndromes (Table 1). The insertion was also discovered in $2.6 \%$ of our controls $(n=4 / 156)$. The p.A75T variant was only identified in $0.2 \%$ of the cohort $(n=1 / 494)$ and was not found in controls $(\mathrm{n}=0 / 139)$. Although this variant is predicted 'probably damaging' $($ score $=0.977)$ to the NIPA2 protein by PolyPhen-2 (http://genetics.bwh.harvard.edu/pph2/), it has a low Grantham score of 58 and is present in $0.2 \%$ of the 6500 individuals on the Exome Variant Server (http://genetics.bwh.harvard.edu/pph2/). The p.A171V variant was detected in $0.64 \%$ of controls $(\mathrm{n}=1 / 156)$, and not in any patients, and is a reported variant.

Our genotyping results differ from those reported by Jiang and colleagues (Jiang et al. 2012). We did not detect either the p.I178F or p.N244S missense variants discovered in their study, not surprising 
since they found them to be rare in their population. We did find the p.N334_E335insD insertion in our cohort but at very different frequencies - in $1.4 \%$ of our patients and $2.6 \%$ of our controls. Jiang and colleagues found this variant in only $0.003 \%(n=1 / 380)$ of their CAE cases and none of their 700 controls.

There are several potential reasons for this discrepancy. First, there could be a difference in the frequency of this insertion between the Han Chinese and Caucasian populations. Second, we noted in our Sanger sequencing analyses that there is a common 3'UTR deletion (rs78803812) with a minor allele frequency of 20\% on both the Exome Variant Server (approximately two-thirds of the 6500 samples are European American, approximately one-third are African American; full details available online: http://www.ncbi.nlm.nih.gov/projects/gap/cgibin/variable.cgi $?$ study_id=phs000400.v1.p1\&phv=165160\&phd=\&pha=\&pht=2536\&phvf=\&phdf=\& phaf $=\& \mathrm{phtf}=\& \mathrm{dssp}=1 \& \mathrm{consent}=\&$ temp $=1)$ and 1000 Genomes databases (approximately $20 \%$ of the samples are of Asian ancestry, including 12\% Han Chinese, and approximately $20 \%$ are of European ancestry; full details available online: http://www.1000genomes.org/about) that if present can mask the presence of the insertion. Specifically, the p.N334_E335insD insertion is only resolvable when sequencing in the forward direction since the 3'UTR deletion is just upstream of the insertion, and as a result masks the insertion when sequencing in the reverse direction. Thus it is possible that Jiang et al missed calling this variant in some of their cases and controls.

The p.A75T and p.A171V variants we discovered are unlikely to be associated with GGE since they are present at low frequency in the general Caucasian population according to the Exome Variant Server or our internal controls, respectively.

Our results suggest, at least in the Caucasian population, that variation in the NIPA2 gene is not 
associated with genetic generalized epilepsy.

\section{ACKNOWLEDGEMENTS}

Elena Aleksoska (Epilepsy Research Centre, University of Melbourne) is acknowledged for performing genomic DNA extractions. This study was supported by National Health and Medical Research Council Program Grant (628952) to S.F.B and I.E.S, an Australia Fellowship (466671) to S.F.B, a Practitioner Fellowship (1006110) to I.E.S and a Postdoctoral Training Fellowship (546493) to M.S.H.

\section{DISCLOSURE OF CONFLICTS OF INTEREST}

None of the authors has any conflict of interest to disclose.

\section{REFERENCES}

Jiang Y, Zhang Y, Zhang P, Sang T, Zhang F, Ji T, Huang Q, Xie H, Du R, Cai B, Zhao H, Wang J, Wu Y, Wu H, Xu K, Liu X, Chan P, Wu X (2012) NIPA2 located in 15q11.2 is mutated in patients with childhood absence epilepsy. Hum Genet. 131: 1217-24. doi: 10.1007/s00439012-1149-3. Epub 2012 Feb 26. 
TABLE 1

Rare NIPA2 variants identified in our study

\begin{tabular}{|l|l|l|l|l|l|l|l|}
\hline Patients & Controls & Location & Type & DNA Level & Protein Level & SNP ID $^{\text {a }}$ & Phenotype $^{\text {rs }}$ \\
\hline $1 / 494$ & $0 / 138$ & Coding & Missense & c.223G>A & p.A75T & rs 150146701 & JME \\
\hline $0 / 494$ & $1 / 156$ & Coding & Missense & c.512C>T & p.A171V & COSM959851 & None \\
\hline $7 / 494$ & $4 / 156$ & Coding & Insertion & c.1002_1003insGAT & p.N334_335EinsD & Novel $^{\text {CAE } ; \text { GTCS }^{c} ; \text { JAE }^{\mathrm{d}} ;}$ \\
\hline
\end{tabular}

${ }^{\mathrm{a}}$ single nucleotide polymorphism identification ID number

${ }^{\mathrm{b}} \mathrm{CAE}$, childhood absence epilepsy

${ }^{\mathrm{c}}$ GTCS, generalized tonic clonic seizures

${ }^{\mathrm{d}}$ JAE, juvenile absence epilepsy

${ }^{\mathrm{e}} \mathrm{JME}$, juvenile myoclonic epilepsy 